\title{
Klienfelter Syndrome Presenting as a Lifelong Anejaculation - A Case Report and Literature Review
}

\author{
Nader Salama* \\ Department of Urology, Alexandria Faculty of Medicine, Alexandria, Egypt \\ *Corresponding author: nadersalama58@yahoo.com
}

Received October 18, 2014; Revised October 31, 2014; Accepted November 03, 2014

\begin{abstract}
Introduction: Klinefelter syndrome (KS) is the most common sex chromosomal anomaly with hypogonadism being a common feature in this syndrome. The ejaculate volume is about normal to low in men with this syndrome. In the present report, we describe the successful treatment of a lifelong anejaculation in a man with KS. This represents the first report of successful treatment of anejaculation in KS. Case presentation: A 24-year-old Caucasian man presented with a lifelong history of failure of ejaculation. A diagnostic work-up revealed the existence of KS and administration of human chorionic gonadotrophin restored the ejaculation. Conclusion: This case report confirms further the increased prevalence of ejaculatory disorders among men with KS in whom associated hypogonadism should be considered a cause of anejaculation. Diagnosis of KS is often delayed. Early diagnosis of the syndrome before puberty is highly recommended and desirable to maintain quality of life.
\end{abstract}

Keywords: Klinefelter syndrome, hypogonadism, anejaculation, human chorionic gonadotrophin

Cite This Article: Nader Salama, "Klienfelter Syndrome Presenting as a Lifelong Anejaculation - A Case Report and Literature Review.” American Journal of Medical and Biological Research, vol. 2, no. 5 (2014): 118120. doi: 10.12691/ajmbr-2-5-2.

\section{Introduction}

Klinefelter syndrome (KS) is the most common sex chromosomal anomaly. It affects $1-2$ in 1000 men. Hypogonadism is a common feature in this syndrome [1]. Diagnosis is often delayed until after puberty due to the significant inconsistency in clinical presentation. KS is associated with a high risk of sexual dysfunction and reduced ejaculate volume. The ejaculatory dysfunctions have a higher prevalence than that of sexual dysfunctions in KS [2,3]. Literature review did not reveal any patient with KS who had a lifelong anejaculation. In this report, we describe the successful treatment of a case of lifelong anejaculation co-existing with KS.

\section{Case Report}

A 24-year-old Caucasian consulted the Department of Urology for a lifelong history of failure of ejaculation. He had just married a month before presentation, was nonsmoker with no history of using alcohol or any drugs and unremarkable past history. He never ejaculated during masturbation nor experienced any wet dreams, but had good early morning erections. He had erections with fantasy thoughts and admitted a good sexual relationship with his wife with an almost daily but dry sexual intercourse.

Physical examination showed a tall stature $(198 \mathrm{~cm})$ and long arm span $(208 \mathrm{~cm})$ with an upper body segment/lower body segments $=78 / 122=0.64$. His body mass index was 28.1. He had no gynecomastia. His secondary sexual characters were normal. The flaccid penis had a length and mid-circumference of $4.5 \mathrm{~cm}$ and 9 $\mathrm{cm}$ respectively with a stretched length of $11 \mathrm{~cm}$. Both testes were firm in consistency and measured 3-cc using a Prader orchidometer. The vas and epididymis were palpable and normal on both sides. Rectal examination revealed a small prostate and non-palpable seminal vesicles while a transrectal ultrasonography was unremarkable and estimated the prostate to be $17 \mathrm{~g}$.

Hormonal investigations showed that folliclestimulating hormone $=16.1 \mathrm{mIU} / \mathrm{mL}$ (normal 1.5-12), leutinizing hormone $=9.04 \mathrm{mIU} / \mathrm{mL}$ (normal 1.8-12.1), total testosterone $=263 \mathrm{ng} / \mathrm{dL}$ (normal 265-800), free testosterone $=7.48 \mathrm{ng} / \mathrm{dL}$ (normal $2-13.5)$, prolactin = $7.77 \mathrm{ng} / \mathrm{mL}$ (normal 2.7 -13.7) and = inhibin B: $21 \mathrm{pg} / \mathrm{mL}$ (normal 11.5-393). KS was suspected, and karyotyping was requested. The cytological report using trypsin Gband showed $47 \mathrm{XXY}$ karyotype confirming the diagnosis of KS. Human chorionic gonadotrophin (hCG) stimulation test was done using hCG injections (choriomon, IBSA, Egypt, 5000 IU, im, 3 consecutive days) to test for Leydig cell functional reserve and its capability of steroidogenesis [4], and to predict any possible existence of testicular sperms for possible retrieval procedures in the future [5]. The stimulation test resulted in the elevation of total testosterone to $(847 \mathrm{ng} / \mathrm{dL})$ indicating the presence of some testicular tissue with reasonable function. When asked to masturbate for a seminal fluid analysis, the patient was unable to ejaculate. 
Four days later, the patient returned to report that he was able to ejaculate during sexual intercourse. He had also one occasion of wet dreams for the first time in his life. He thought that the ejaculate was of reasonable volume as his clothes and sheets on the bed were soaked with semen. Semen analysis was immediately requested, but the patient was still unable to ejaculate with masturbation. The patient was then given the same hCG preparation; 2 injections per week (5000 IU, each, im). He was able to ejaculate easily during each sexual intercourse, but not during masturbation.

\section{Discussion}

Failure of ejaculation may be caused by diabetes, retroperitoneal lymph node dissection, spinal cord trauma, disseminated sclerosis, transverse myelitis and psychological troubles. Hyperprolactinemia and hypogonadism may also be included among these offending causes [6]. In the present report, our patient suffered from hypergonadotrophic hypogonadism. This was related to his primary testicular failure as a consequence of KS.

$\mathrm{KS}$ is the most common sex chromosomal anomaly resulting from the presence of one or more extra $\mathrm{X}$ chromosomes and affecting 1-2 in 1000 men. It is characterized phenotypically by a tall stature, small firm testes, gynecomastia, abdominal adiposity and associated with hypergonadotrophic hypogonadism. Its commonest karyotype is 47, XXY [1]. Diagnosis is often delayed until after puberty due to the significant inconsistency in clinical presentation [1,3].

The presenting complaint in our index patient was a lifelong inability to ejaculate. Studies investigating the ejaculatory pattern in KS are extremely scarce. Yoshida et al showed 14 (35\%) out of 40 men with KS who perceived low ejaculate volumes [2]. Corona et al reported 23 patients with KS. Among these, 28.6\% and 33.3\% claimed slight and moderate to severe reduction in their ejaculates [3]. However, neither study included any man with complete lack of ejaculation as seen in our patient who did not complain, however, of any sexual disorder. This agrees with Miyagawa et al [7] who indicated that ejaculatory disturbances may occur with higher testosterone level than erectile complaints in patients with hypogonadism.

The exact mechanism of how testosterone influences the process of ejaculation is still lacking. Corona and his colleagues, in one of their studies demonstrating the relation between the different testosterone levels and severities of ejaculatory disturbances, suggested some hypotheses to elucidate the link between testosterone and ejaculation [8]. The first hypothesis is psycho-endocrine which depends on the fact that sexual confidence has an important role in control of ejaculation timing. When this confidence drops with hypogonadism, it would decrease the signals needed to reach orgasm and ejaculation. The second hypothesis is a central neurological. This has been suggested based on the decrease in brain level of serotonin, an important neurotransmitter in delaying ejaculation, following chronic administration of testosterone. With hypogonadism, the reverse occurs, thus delaying ejaculation. The third one credits the nitric oxidephosphodiesterase-5 system with an important role in regulating the motility of male genital system. This system is under the control of testosterone. Therefore, a decline in testosterone will delay ejaculation. The last hyposthesis is mechanical where small ejaculate bolus in cases of hypogonadism may disturb the propulsion mechanics of the accessory genital organs, leading to difficult ejaculation.

The treatment of the index patient involved hCG therapy which stimulates Leydig cell steroidogeneis with the aim of achieving physiological levels of plasma testosterone [4]. This was successful in relieving his symptoms of lifelong anejaculation.

\section{Conclusion}

We managed a rare case of lifelong inability to ejaculate in a patient with KS. This case report corroborates the high prevalence of ejaculatory disorders among men with this syndrome. It reconfirms the significant discrepancy in clinical presentation of the syndrome and its commonly missed diagnosis at an early stage. Men presenting with hypogonadism, particularly if associated with abnormal ejaculate volume and smallsized testes, should be examined thoroughly to rule out the possible existence of $\mathrm{KS}$, which will then require hormone replacement therapy to maintain quality of life.

\section{Acknowledgements}

A written informed consent was obtained from the patient for publication of this case report.

A copy of the written consent is available for review by the Editor-in-Chief of this journal.

This case report manuscript was written without any financial support, except for the author's personal costs.

Statement of authorship: Nader Salama: Conception and design of the study, collection, analysis and interpretation of data, and drafting the article with final approval of its completed form.

\section{Statement of Competing Interests}

The author declares that he has no competing interests.

\section{List of Abbreviations}

KS: Klienfelter syndrome; hCG: Human Chorionic Gonadotrophin; mTESE: Microsurgical testicular sperm extraction

\section{References}

[1] Morris, J. K., Alberman, E., Scott, C. and Jacobs, P, "Is the prevalence of Klinefelter syndrome increasing?”, Eur J Hum Genet, 16, 163-170, 2008.

[2] Yoshida, A., Miura, K., Nagao, K., Hara, H., Ishii, N. and Shirai, $M$, "Sexual function and clinical features of patients with Klinefelter's syndrome with the chief complaint of male infertility”, Int J Androl, 20, 80-85, 1997.

[3] Corona, G., Petrone, L., Paggi, F., Lotti, F., Boddi, V., Fisher, A., Vignozzi, L., Balercia, G., Sforza, A., Forti, G., Mannucci, E. and 
Maggi, M, "Sexual dysfunction in subjects with Klinefelter's syndrome”, Int J Androl, 33, 574-580, 2010.

[4] Fukutani, K., Ishida, H., Shinohara, M., Minowada, S., Niijima, T. and Isurugi, K, "Responses of serum testosterone levels to human chorionic gonadotrophin stimulation in patients with Klinefelter's syndrome after long-term androgen replacement therapy”, Int $J$ Androl, 6, 5-11, 1983.

[5] Madgar. I, Dor. J, Weissenberg. R, Raviv. G, Menashe. Y and Levron J, "Prognostic value of the clinical and laboratory evaluation in patients with nonmosaic Klinefelter syndrome who are receiving assisted reproductive therapy”, Fertil Steril, 77, 1167-1169, 2002.
[6] Witt, M.A. and Grantmyre, J.E, “Ejaculatory failure”, World $J$ Urol, 11, 89-95, 1993.

[7] Miyagawa, Y., Tsujimura, A., Matsumiya, K., Takao, T., Tohda, A., Koga, M., Takeyama, M., Fujioka, H., Takada, S., Koide, T. and Okuyama, A, "Outcome of gonadotropin therapy for male hypogonadotropic hypogonadism at university affiliated male infertility centers: a 30-year retrospective study", J Urol, 173, 2072-2075, 2005

[8] Corona, G., Jannini, E.A., Mannucci, E., Fisher, A.D., Lotti, F., Petrone, L., Balercia, G., Bandini, E., Chiarini, V., Forti, G. and Maggi, M, "Different testosterone levels are associated with ejaculatory dysfunction”, J Sex Med, 5, 1991-1998, 2008. 